\title{
Evaluation of heart rate measurements in clinical studies: a prospective cohort study in patients with heart disease
}

\author{
Marco Albanese $^{1,3} \cdot$ Michaelis Neofytou $^{1}$ - Taoufik Ouarrak ${ }^{2} \cdot$ Steffen Schneider $^{2}$ • \\ Wolfgang Schöls ${ }^{1}$
}

Received: 31 December 2014 / Accepted: 15 March 2016/Published online: 29 March 2016

(C) The Author(s) 2016. This article is published with open access at Springerlink.com

\begin{abstract}
Purpose The purpose of this study was to evaluate the measurement of heart rate undertaken in clinical studies by (1) assessing the repeatability and reproducibility of heart rate measurements by various methods and under various conditions and (2) determining whether a single heart rate measurement at rest is representative of the circadian and inter-day variation of heart rate. Methods Prospective cohort study in 102 patients with various types of heart disease at Duisburg Heart Center, Germany between 2011 and 2012. The heart rate measurements were based on self-assessment, ECG tracings at rest, and bicycle stress ECG in the office as well as 24-h Holter ECG.

Results Office measurements and self-assessment at rest as well as 24-h Holter ECG and self-assessment at rest are highly correlated, but no correlation between self-assessment and office recordings $/ 24 \mathrm{~h}$ recordings under exercise conditions was seen. Coefficient of variability was below $10 \%$ for the self-assessment and for office measurements at rest. There were no differences in coefficient of variability during the day and within the 6 days for self-assessment of heart rate at rest and circadian variation was normal.

Conclusions At rest heart rate measurements by various methods agree sufficiently and inter-day/circadian variation is adequately represented. Under exercise conditions self-
\end{abstract}

Marco Albanese

malbanes@bluewin.ch

1 Herzzentrum Duisburg, Gerrickstr. 21, 47137 Duisburg, Germany

2 Stiftung Institut für Herzinfarktforschung, Bremserstraße 79 - Haus M, 67063 Ludwigshafen am Rhein, Germany

3 Present address: Regionalspital Surselva, Spitalstr. 6, 7130 Ilanz, Switzerland assessment of heart rate is not valuable and use of $24 \mathrm{~h}$ Holter as well as stress ECG recordings is necessary. Thus, self-reported heart rate measurements by the patient at rest seem to be reliable, but should be used in clinical studies only for heart rate assessment at rest.

Keywords Heart rate variability $\cdot$ Heart rate measurement . Pharmacological intervention studies $\cdot$ Clinical studies $\cdot$ Heart disease

\section{Introduction}

Previous epidemiological studies generated evidence for a correlation between heart rate and risk of cardiovascular disease and hence life expectancy in the general population and in patients with coronary heart disease (CHD) [1-4]. Most of coronary perfusion takes place in diastole, a phase characterized by a reduced wall tension compared to systole. Prolonging diastole should improve coronary perfusion and might thus decrease CHD morbidity and mortality [5]. Accordingly, several studies have addressed the effect of heart rate lowering drugs on symptoms and prognosis of patients with heart failure and $\mathrm{CHD}[6,7]$.

In these studies, dose titration of heart rate lowering agents was primarily based on measurements of heart rate in ECG tracings at rest, occasionally on 24-h Holter recordings as well. Some studies simply relied on pulse counts obtained by the patients.

The setting for the ECG recording was not specifically defined or described in the study protocols or other publications [8]. Furthermore, a correlation of ECG derived parameters with "real world" measurements of heart rate by the patient during his daily activities has never been performed. There is also some uncertainty on the variability of heart rate measurements within the day and from day to day. Finally, 
with respect to pathophysiology, the question arises how well a single heart rate measurement at rest reflects the heart rate pattern during the whole day. The lack of information on all these issues sheds some doubt on the significance of single resting heart rate measurements suggested by respective studies.

We therefore analyzed heart rate measurements obtained with various methods and at various points in time. Our primary objectives were (1) to assess the repeatability and reproducibility of heart rate measurements by various methods and under various conditions and (2) to determine whether a single, casual heart rate measurement at rest is representative of the heart rate pattern during the day and between six consecutive days. Furthermore, data collected might shed light on the issue, whether self-reported heart rate measurements by the patient are reliable and, therefore, can be used for heart rate assessment in clinical studies.

\section{Methods}

\section{Study patients and data collection}

This investigation was a single center, prospective cohort study in 102 patients aged $42-80$ years with various types of heart disease treated according to ESC and National Guidelines [9, 10]. Patient screening was done in the hospital and data sampling was continued upon discharge from the hospital. Independent of the underlying heart disease, eligible patients had an indication for heart rate lowering therapy (CAD and heart failure) and received all drugs according to standard recommendations. The focus was, however, not on specific therapies, but on the assessment of heart rate under various conditions. Heart rate was determined according to protocol by the patient himself, and by study personnel blinded for the patient measurements, based on resting ECGs, Holter recordings, and exercise test ECG tracings. For the complete protocol, please refer to the online-publication.

\section{Definitions}

Variability in heart rate during the day and day to day variability were analyzed and compared to the heart rate recorded by the ECG at rest in the clinic (office) based on coefficient of variability, repeatability, and reproducibility. Change and reproducibility provide values of the upper limits for the variation with high probability (that is for the difference between repeated measurements). In order to compare self-assessments and the heart rate recorded by the ECG at rest in the clinic, Holter recordings and stress ECG, heart rates agreement was used and defined as a deviation of less than 10 beats/min from mean. For the visual analysis of the 24-h heart rate pattern, we defined a tachycardia period as the duration in time of heart rates deviation of more than 10 beats/min from mean.

\section{Data analysis}

Measurements recorded during the ambulatory period were stored on a personal computer and screened for artifacts as defined by previously described criteria [11]. Only recordings with less than $20 \%$ error measurements were accepted for evaluation. Regarding self-assessment, only daytime values were available.

\section{Statistical analysis}

Categorical variables are described by using percentages and absolute numbers, continuous variables by using mean, standard deviation, and range. The Brevais-Pearson coefficient was used for correlations, and reproducibility was assessed using the Bland-Altman approach [12]. Change between duplicate recordings was calculated by subtracting the first from the second recording. A comparison between baseline and repeat recording was done by using the $t$ test. Consistency was obtained by calculating the difference between baseline and repeat recordings, disregarding the sign of the difference.

Repeatability was defined as twice the standard deviation of the changes between two repeated recordings. To compare the reproducibility of different variables, the maximal biological variation (MBV) of the variables was calculated (twice the standard deviation of the between-measurement differences, divided by four times the standard deviation of the two duplicate recordings) [13]. All statistical calculation was performed using SAS 9.3.

\section{Results}

\section{Clinical and treatment characteristics}

The clinical characteristics of the patient population are shown in Table 1. The proportion of patients with hyperlipidemia, hypertension, smoking habits, peripheral arterial obstructive disease, or diabetes mellitus reflected a typical population with heart disease. Almost all were on heart rate lowering medication and most of them had only a slight reduction in ejection fraction. Most performed moderate physical activity in terms of exercise intensity defined by the MET concept at some point of the day. Nearly half of the patients exposed themselves to heavy physical activity striving for cardiovascular fitness and the heart frequency range observed in this study is therefore comparable to that one of a normal, healthy population of the same age range. Exercise levels of peak physical activities at home and during bicycle stress ECG test expressed in MET were comparable in $72 \%$ of patients (data not shown).

According to the protocol, we expected the patients to record six measurements at rest during the day for a total of 
Table 1 Base line characteristics of patients included in study

\begin{tabular}{|c|c|}
\hline \multicolumn{2}{|l|}{ Demographics } \\
\hline Total, $\%$ & $100(102)$ \\
\hline Female, $\%$ & $29.4(30 / 102)$ \\
\hline Age, (years) mean $( \pm \mathrm{SD})$ : & $63.2( \pm 10.43)$ \\
\hline Age $<50, \%$ & $10.8(11 / 102)$ \\
\hline Age $50-70, \%$ & $60.8(62 / 102)$ \\
\hline Age $>70, \%$ & $28.4(29 / 102)$ \\
\hline \multicolumn{2}{|l|}{ Clinical presentation } \\
\hline BMI, $\mathrm{kg} / \mathrm{m}^{2}$ mean $( \pm \mathrm{SD})$ : & $27.5( \pm 3.6)$ \\
\hline $\mathrm{BMI}>30, \%$ & $19.6(20 / 102)$ \\
\hline \multicolumn{2}{|l|}{ Medical history ${ }^{*}$} \\
\hline Coronary heart disease, $\%$ & $75.2(76 / 101)$ \\
\hline Hypertension, $\%$ & $91.1(91 / 101)$ \\
\hline Hypercholesterolemia, \% & $54.5(55 / 101)$ \\
\hline Diabetes mellitus, $\%$ & $12.9(13 / 101)$ \\
\hline $\mathrm{AF}, \%$ & $14.9(15 / 101)$ \\
\hline \multicolumn{2}{|l|}{ Ejection fraction } \\
\hline Slight reduction (EF 40-60\%), \% & $27.7(28 / 101)$ \\
\hline Moderate reduction (EF $30-40 \%$ ), \% & $8.9(9 / 101)$ \\
\hline Severe reduction $(\mathrm{EF}<30 \%), \%$ & $3.9(4 / 101)$ \\
\hline Contraindication to $ß$-Blockade, $\%$ & $6.9(7 / 101)$ \\
\hline \multicolumn{2}{|l|}{ Medication* } \\
\hline Heart rate lowering agents, $\%$ & $99.0(101 / 102)$ \\
\hline Diuretics, $\%$ & $54.9(56 / 102)$ \\
\hline Antihypertensive medication, $\%$ & $77.5(79 / 102)$ \\
\hline Statins, $\%$ & $75.5(77 / 102)$ \\
\hline Inhibition of platelet function and anticoagulation, $\%$ & $95.1(97 / 102)$ \\
\hline Thyroid hormones & $8.8(9 / 102)$ \\
\hline \multicolumn{2}{|c|}{ Classification of physical activity in terms of exercise intensity* } \\
\hline Light, $\%$ & $77.5(79 / 102)$ \\
\hline Moderate, $\%$ & $92.2(94 / 102)$ \\
\hline Heavy, \% & $47.1(48 / 102)$ \\
\hline Very heavy, \% & $20.6(21 / 102)$ \\
\hline Unduly heavy, \% & $3.9(4 / 102)$ \\
\hline
\end{tabular}

$B M I$ body mass index, $S D$ standard deviation, $A F$ atrial fibrillation, $E F$ ejection fraction

*Multiple entries possible

6 days and three measurements per day under exercise conditions. For consistency reasons, we used only selfmeasurement of heart rate with corresponding Holter recordings and office treadmill heart rate measurements for statistical analyses. Therefore, the number of observations read for the analysis of heart at rest assessed by self-assessment was 3559 in total and 3474 were used. Similarly, for the comparison of heart rate under exercise conditions assessed by self-assessment, the number of observations read was 1831 in total and 1620 were used. Heart rates measured under various conditions are reported in Table 2. Mean heart rate at rest was below 70 beats $/ \mathrm{min}$. As expected, standard deviations of the mean
Table 2 Mean (SD), minimum, and maximum values of heart rates measured under various conditions

\begin{tabular}{llll}
\hline Period & Mean & SD & Range \\
\hline HR office at rest $^{\mathrm{a}}$ & 67.4 & 10.1 & $45-98$ \\
HR office treadmill maximum $^{\mathrm{b}}$ & 118.6 & 19.2 & $68-164$ \\
HR 24 h all values & 69.5 & 8.7 & $47-95$ \\
HR 24 h minimum & 51.5 & 8.3 & $35-93$ \\
HR 24 h maximum & 113.5 & 17.9 & $80-172$ \\
HR self all values & 66.6 & 9.8 & $48-105$ \\
HR self minimum & 57.0 & 11.1 & $46-96$ \\
HR self maximum & 88.6 & 16.1 & $60-146$ \\
\hline
\end{tabular}

$H R$ heart rate, $H R$ office at rest $\mathrm{HR}$ derived ECG recordings at rest in the clinic, HR $24 h$ HR derived from 24-h Holter recording, HR self $\mathrm{HR}$ derived from self-assessment; $H R$ office treadmill $H R$ derived from a bicycle ECG stress tests, $S D$ standard deviation expressed as beats/min

${ }^{\text {a }}$ Mean of 3 ECG recordings at rest

${ }^{\mathrm{b}}$ Recorded mean of 24-h Holter recording

${ }^{\mathrm{c}}$ Mean of all values measured by patients

heart rate were higher under exercise conditions. Correlation coefficients between office measurements and self-assessment at rest as well as 24-h Holter ECG and self-assessment at rest are shown in Table 3. Correlation coefficients between office measurements and self-assessment at rest as well as 24-h Holter ECG and self-assessment at rest were similar and much higher than any correlation under exercise conditions. In fact, looking at the correlation coefficients for exercise measurements, there was only a very weak association between office measurements and those based on self-assessment.

Change, consistency, repeatability, mean biological variability (MBV), and coefficient of variability for heart rate

Table 3 Mean values $( \pm \mathrm{SD})$ and correlation coefficients (CC) between values of heart rate measured under various conditions in the whole study population

\begin{tabular}{llll}
\hline Period & \multicolumn{2}{l}{ Mean values $( \pm \mathrm{SD})$} & $\mathrm{CC}$ \\
\hline HR office at rest & 67.4 & 10.1 & 0.68 \\
HR self all values & 66.6 & 9.8 & \\
HR office treadmill maximum & 118.6 & 19.2 & 0.17 \\
HR self maximum & 88.6 & 16.1 & \\
HR 24 h all values & 69.5 & 8.7 & 0.67 \\
HR self all values & 66.6 & 9.8 & \\
HR 24 h minimum & 51.5 & 8.3 & 0.57 \\
HR self minimum & 57.0 & 11.1 & \\
HR 24 h maximum & 113.5 & 17.9 & 0.26 \\
HR self maximum & 88.6 & 16.1 & \\
\hline
\end{tabular}

$H R$ heart rate, $H R$ office at rest $\mathrm{HR}$ derived ECG recordings at rest in the clinic, HR $24 h$ HR derived from 24-h Holter recording, HR self HR derived from self-assessment, HR office treadmill HR derived from a bicycle ECG stress tests 
Table 4 Reproducibility indexes for heart rate measured at rest in the office and with selfassessment by patients

\begin{tabular}{lllllll}
\hline Period & Change & $p$ value & Consistency & Repeatability & MBV (\%) & CV \\
\hline HR office at rest & -2.31 & 0.0046 & 7.6 & 14.5 & 59 & 9.3 \\
HR self all values & -0.06 & 0.4777 & 5.9 & 15.1 & 66 & 7.4 \\
HR self morning values & -0.09 & 0.2956 & 5.5 & 14.5 & 72 & 7.6 \\
HR self midday values & -0.07 & 0.5296 & 5.8 & 16.0 & 73 & 8.1 \\
HR self night values & -0.09 & 0.0499 & 5.3 & 13.1 & 71 & 7.5 \\
\hline
\end{tabular}

$H R$ heart rate, $H R$ office at rest $\mathrm{HR}$ derived $\mathrm{ECG}$ recordings at rest in the clinic, $H R$ self $\mathrm{HR}$ derived from selfassessment, $M B V$ mean biological variability, $C V$ coefficient of variability measured at rest in the office and with self-assessment are reported in Table 4. Consistency and coefficient of variability indicated a greater variation for office measurements at rest than for self-assessment. For all measurements, the coefficient of variability was below the acceptable value of $10 \%$ and reproducibility was modest. Within self-assessment data, the change was very small but as would have been expected the MBV was higher. Repeatability was worse for the heart rate variability within a day as well as for the heart rate variability within different days during the 12 o'clock self-assessment at rest. Looking at heart variability of the self-assessment at rest during the day, there were no differences in coefficient of variability during the day (HR self all day) and within the 6 days of self-assessment analyzing the different time points of measurement except for the midday measurement. Analyzing repeatability indices in subgroups of patients with reduced ejection fraction or with different intensities of physical exercise, these indexes were worse, but with no relevant differences in repeatability (data not shown).

Due to the study design (only one 24-h Holter recording was performed), we used heart rates agreement defined as a deviation of less than 10 beats/min from mean in order to compare heart rates under the various conditions. Maximum heart rates in Holter recordings during daytime will correspond to exercise in a patient under adequate heart rate controlling medication and minimum heart rate will correspond to rest (Table 2). Therefore, it was possible to compare heart rates under exercise (office stress ECG and Holter recordings) and at rest (ECG at rest in the clinic and Holter recordings) to heart rates of self-assessment (Table 5). Comparing heart rates of office stress ECG vs. heart rates of self-assessment under exercise conditions, only $10 \%$ of the measurements showed an agreement with heart rate determined by self-assessment. The same holds true for the comparison of maximum heart rates documented by the Holter recording during the daytime with heart rates of self-assessment under exercise. In this case, only $9 \%$ of patients showed comparable heart rates to the self-assessment. Comparison of the mean heart rate during the Holter recording with the mean heart rate of the self-assessment showed a better agreement with $80 \%$ of the measurements being comparable to the heart rate determined by self-assessment. Similar results were obtained comparing the minimum heart rate of the Holter recording with the minimum heart rate of the self-assessment with $76 \%$ of the measurements showing an agreement.

Analyzing the 24-h heart rate pattern for tachycardia periods, we observed the following:

1) $60 \%$ of patients showed tachycardia periods with a duration of less than $25 \%$ of the time recorded (range 0 $24.3 \%$, mean $15.9 \%$ ) during the $24 \mathrm{~h}$,

2) $38 \%$ of patients presented tachycardia periods with a duration of 25 to $50 \%$ of the time recorded (range $25.3-44.6 \%$, mean $32.1 \%$ ) during the $24 \mathrm{~h}$ and,

3 ) only $2 \%$ of patients had tachycardia periods with a duration of 50-75\% of the time recorded (range 57.6-63.6\%, mean $60.6 \%$ ) during the $24 \mathrm{~h}$. During the sleeping hours, the registered tachycardia periods encompassed $12.4 \%$ of the time recorded (range 0-52.2\%).

The study included 15 patients with atrial fibrillation. All of them were already diagnosed with atrial fibrillation and no newly diagnosed atrial fibrillation was detected. Twenty percent $(3 / 15)$ presented with permanent fibrillation and $80 \%$ $(12 / 15)$ with paroxysmal fibrillation. In the patients with paroxysmal fibrillation, 9/12 patients showed sinus rhythm, 2/12 patients had permanent fibrillation, and 1/12 patients showed only short phases of atrial fibrillation on 24-h Holter recordings. Analyzing the 24-h heart rate pattern for tachycardia periods, we observed the following: (1) $7 / 15$ patients showed

Table 5 Agreement between self-assessment and Holter recordings and office treadmill heart rate measurements

Patients with HR comparable to self-assessment

\begin{tabular}{ll}
\hline HR office treadmill max & $10 \%$ \\
HR 24 h mean & $80 \%$ \\
HR 24 h min & $76 \%$ \\
HR 24 h max & $9 \%$ \\
\hline
\end{tabular}

Proportion (\%) of patients showing comparable heart rates with the selfassessment are depicted

$H R$ heart rate, $H R$ office treadmill $\mathrm{HR}$ derived from a bicycle ECG stress tests, $H R 24 h$ HR derived from 24-h Holter recording, $H R$ self $\mathrm{HR}$ derived from self-assessment 
tachycardia periods with a duration of less than $25 \%$ of the time recorded (range 7.5-23.6 \%, mean $15.5 \%$ ) during the $24 \mathrm{~h}$, (2) 7/15 patients presented tachycardia periods with a duration of 25 to $50 \%$ of the time recorded (range $27.2-$ $37.0 \%$, mean $34.8 \%$ ) during the $24 \mathrm{~h}$, and (3) only $1 / 15$ patients had tachycardia periods with a duration of 50-75\% of the time recorded (range $56.7 \%$ ) during the $24 \mathrm{~h}$. In the subgroup of the three patients with permanent atrial fibrillation $2 / 3$ patients showed tachycardia periods with a duration of less than $25 \%$ of the time recorded and $1 / 3$ patients tachycardia periods with a duration of 25 to $50 \%$ of the time recorded. In the subgroup of patients with paroxysmal atrial fibrillation, 6/12 patients showed tachycardia periods with a duration of less than $25 \%$ of the time recorded and $5 / 12$ patients tachycardia periods with a duration of 25 to $50 \%$ of the time recorded and $1 / 12$ patients had tachycardia periods with a duration of $50-75 \%$ of the time recorded.

\section{Discussion}

\section{Key findings}

We hypothesized that the measurement of heart rate undertaken in clinical studies was not representative of (1) the circadian and inter-day variation and (2) of the exercise-induced variation of heart rate during the patient's everyday life.

Our hypothesis was only partially confirmed and we could show that the exercise-induced variation of heart rate during the patient's everyday life is not adequately represented in clinical studies. Instead self-reported heart rate measurements at rest represent adequately the inter-day and circadian variation of heart rate. Self-reported heart rate measurements by the patient at rest are thus reliable, but can be used in clinical studies only for heart rate assessment at rest.

\section{Study strength and limitations}

Major part of this study is a measurement method comparison. The statistical aspects of measurement method comparisons have been thoroughly discussed by Altman and Bland [12, 14]. According to their findings, the comparison of mean values by significance testing is inappropriate because a nonsignificant result might occur as result of both a large measurement error and agreement on the average. The calculation of the correlation coefficient is another often misused method because it is not a measure of agreement, but of association. For this reason, we used the coefficient of variability that has the advantage of indicating the relation between the magnitude of observations and their variations. In addition, the use of the coefficient of variation allows for comparison between different methods and different groups of subjects.
Next, defining what is the normal resting heart rate and what is an acceptable deviation is troublesome. We based our decision on the following studies. First, in a study on resting heart rate in Diabetics with altered autonomic function, a standard deviation (SD) of to 10 beats/min was noticed in the healthy comparison group [15]. In a much larger study by Erikssen and Rodahl in 2014 apparently healthy middleaged men aged 40-59 years, the mean heart rate at rest was 61 beats/min and the observed SD was 9.7 beats/min [16]. In this study, self-assessment was compared to auscultation and resting ECGs. In another publication by Palatini et al. [11] on the reproducibility of heart rate measurement in the clinic and with $24 \mathrm{~h}$ intermittent recorders, a SD of up to 9.6 beats/min was accepted. In one of the larger pharmacological intervention studies - the BEAUTIFUL study - a SD of 8.7 beats/min from mean was reported $[5,7]$. Based on these studies, we considered a deviation of more than 10 beats/min from mean to be relevant.

Another limit of this study is the relatively small number of patients included in the study, but this is counterbalanced by the high number of observations used. Furthermore, we did not have a normal control group. However, this is not interfering with the objective of this study focused on the evaluation of heart rate measurement undertaken in clinical studies.

\section{Comparison with other studies and interpretation of results}

As to our knowledge, there are no published studies on this argument and therefore comparison with the results of others may be only indirect and limited.

In healthy individuals, there is a circadian pattern of heart rate with a peak heart rate around $10 \mathrm{am}$, and a gradual decline during the night with a nadir just prior to awakening [17]. Disease states such as congestive heart failure with alterations in autonomic tone and medications can have an effect on circadian rhythms [18]. The healthy controls in two studies on abnormalities in ambulatory $24 \mathrm{~h}$ heart rate in diabetes and in inappropriate sinus tachycardia showed tachycardia periods spanning 29 and $42 \%$ of the registered $24 \mathrm{~h}$, respectively. No tachycardia periods occurred during the sleeping hours $[19$, 20]. This heart rate pattern was not changed in our population on heart rate lowering therapy except for the presence of tachycardia periods during the sleeping hours. Furthermore, there were no differences in coefficient of variability during the day and within the 6 days for self-assessment of heart rate at rest. Due to the low numbers and missing follow-up evaluation of a possible prognostic, significance of these tachycardia periods during the night is not possible. In addition, selfassessment with acquisition of about 36 values per patients as a method of measurement is only slightly superior to three ECG recordings at rest in evaluating resting heart rate. It is noteworthy that the coefficients of variability of both methods 
are with a value of less than $10 \%$ in an acceptable range. This confirms previous data that heart rate recorded over the $24 \mathrm{~h}$ has a better reproducibility than office heart rate and may thus be a better prognostic indicator than traditional measurement of resting heart rate in the hospital setting [11]. A possible reason for the higher statistical correlations in this study with respect to other studies comprising healthy subjects maybe the use of heart rate controlling medication [11].

Furthermore, atrial fibrillation might have an impact on the reproducibility of the various heart rate measurements. According to our results, atrial fibrillation was not a confounding factor due to fact that most patients in this study had only paroxysmal fibrillation with a majority of patients still showing sinus rhythm. In addition, analysis of the 24-h heart rate pattern for tachycardia periods showed that the results of patients with atrial fibrillation were comparable to patients with sinus rhythm.

With regard to heart rate assessment under exercise or stress conditions, self-assessment underestimates the maximum heart rate patients are exposed to. This seems to be supported by the fact that peak exercise levels at home and during bicycle stress ECG were similar in a majority of patients. Thus, from a methodological point of view, bicycle exercise ECG as well as 24-h Holter recordings are more precise in documenting the maximum heart rates reached. Reasons for the above observation might be (1) the time delay between exercise and self-measurement and (2) the fact that the patients may simply have omitted the self-assessment in certain situations.

Our study suggests that there is no pronounced variability of heart rate measurements taken at various time points neither during the day or at different days and, therefore, physiological variation in heart rate does not seem to be a confounding factor in the conduction of pharmacological intervention studies. With regard to the recording of the maximum heart rate under exercise or stress conditions self-assessment of heart rate is not a valuable neither a trustworthy method of investigation. Furthermore, heart rates registered point to relevant tachycardia under exercise or stress conditions. Noticeably in none of the intervention studies published so far, the maximum heart rate on heart rate limiting medication was documented. Based on the results of this study, we would recommend future studies to address the question, whether the extent of the heart rate limiting effect under exercise or stress conditions influences the end points such as cardiovascular morbidity and mortality.

\section{Conclusions}

Dose titration of heart rate lowering agents in pharmacological intervention studies is actually based mostly on measurements of heart rate in ECG tracings at rest. In our study, heart rate measurements at rest in the office and based on selfassessment are comparable and reproducible. Physiological variation in heart rate does not seem to be a confounding factor in the conduction of pharmacological intervention studies. But under exercise conditions, heart rate measurements taken in the office and by 24-h Holter monitoring do not agree in a sufficient manor with measurements based on selfassessment.

Consequently, self-assessment of heart rate provides the same information as conventional methods of measurement at rest. Under exercise conditions, the routine use of 24-h Holter as well as stress ECG recordings is helpful for a more complete data acquisition. Therefore, (1) self-reported heart rate measurements by the patient seem to be reliable only at rest and, therefore, should be used in clinical studies only for heart rate assessment at rest and (2) it seems reasonable to propose a standardized operating procedure for heart rate measurements in clinical studies comprising ECG tracings at rest during every visit (a minimum of three), three treadmill ECG, and three 24-h Holter recordings. Such common standardized operating procedure may enable the scientific community to compare more easily clinical studies without being limited by different methodological designs.

Acknowledgments This study was funded by a grant from SERVIER Deutschland GmbH. Servier has no influence on this study in any aspect including study design, interpretation of the data, results, or publication. We thank Dirk Böhm for creating the data bank and Dunja Schulenburg for help with the patients.

Author's contribution Marco Albanese was responsible for the conception and design of the study, as well as interpretation of the data and conclusions drawn. M. Albanese also drafted the manuscript. Michaelis Neofytou collected and analyzed the data. Taoufik Ouarrak performed statistical analysis of the data. Steffen Schneider and Wolfgang Schoels examined and revised the manuscript for intellectual content and gave final approval of the manuscript submitted. There is no conflict of interest for any of the authors. Marco Albanese is the corresponding author.

Open Access This article is distributed under the terms of the Creative Commons Attribution 4.0 International License (http:// creativecommons.org/licenses/by/4.0/), which permits unrestricted use, distribution, and reproduction in any medium, provided you give appropriate credit to the original author(s) and the source, provide a link to the Creative Commons license, and indicate if changes were made.

\section{References}

1. Kannel WB, Kannel C, Paffenbarger RS Jr, Cupples LA (1987) Heart rate and cardiovascular mortality: the Framingham study. Am Heart J 113(6):1489-1494

2. Reunanen A, Karjalainen J, Ristola P, Heliovaara M, Knekt P, Aromaa A (2000) Heart rate and mortality. J Intern Med 247(2): 231-239

3. Diaz A, Bourassa MG, Guertin MC, Tardif JC (2005) Long-term prognostic value of resting heart rate in patients with suspected or proven coronary artery disease. Eur Heart J 26(10):967-974 
4. Hjalmarson A, Gilpin EA, Kjekshus J, Schieman G, Nicod P, Henning H, Ross J Jr (1990) Influence of heart rate on mortality after acute myocardial infarction. Am J Cardiol 65(9):547-553

5. Fox K, Ford I, Steg PG, Tendera M, Robertson M, Ferrari R (2008) Heart rate as a prognostic risk factor in patients with coronary artery disease and left-ventricular systolic dysfunction (BEAUTIFUL): a subgroup analysis of a randomised controlled trial. Lancet 372(9641):817-821

6. Poole-Wilson PA, Cleland JG, Di Lenarda A, Hanrath P, Komajda M, Metra M, J Remme W, Swedberg K, Torp-Pedersen C (2002) Rationale and design of the carvedilol or metoprolol European trial in patients with chronic heart failure: COMET. Eur J Heart Fail 4(3):321-329

7. Fox K, Ford I, Ford SPG, Tendera M, Ferrari R (2008) Ivabradine for patients with stable coronary artery disease and left-ventricular systolic dysfunction (BEAUTIFUL): a randomised, double-blind, placebo-controlled trial. Lancet 372(9641):807-816

8. Vogel C, Wolpert C, Wehling M (2004) How to measure heart rate? Eur J Clin Pharmacol 60(7):461-466

9. www.escardio.org/Guidelines-\&-Education/Clinical-PracticeGuidelines/listing. In: ed

10. www.leitlinien.dgk.org. In: ed

11. Palatini P, Winnicki M, Santonastaso M, De Venuto G, Zanata G, Bertolo O, Frigo G, Pessina AC (2000) Reproducibility of heart rate measured in the clinic and with 24-h intermittent recorders. Am J Hypertens 13(1 Pt 1):92-98
12. Bland JM, Altman DG (1986) Statistical methods for assessing agreement between two methods of clinical measurement. Lancet 1(8476):307-310

13. Thijs L, Amery A, Clement D, Cox J, de Cort P, Fagard R, Fowler G, Guo C, Mancia G, Marin R (1992) Ambulatory blood pressure monitoring in elderly patients with isolated systolic hypertension. $\mathrm{J}$ Hypertens 10(7):693-699

14. Altman DG, Bland JM (1983) Measurement in medicine: the analysis of method comparison studies. Statistician 32:307-317

15. Ewing DJ, Campbell IW, Clarke BF (1981) Heart rate changes in diabetes mellitus. Lancet 1(8213):183-186

16. Erikssen J, Rodahl K (1979) Resting heart rate in apparently healthy middle-aged men. Eur J Appl Physiol Occup Physiol 42(1):61-69

17. Millar-Craig MW, Bishop CN, Raftery EB (1978) Circadian variation of blood-pressure. Lancet 1(8068):795-797

18. Panina G, Khot Un, Nunziata E, Cody RJ, Binkley PF (1995) Assessment of autonomic tone over a 24-h period in patients with congestive heart failure: relation between mean heart rate and measures of heart rate variability. Am Heart J 129(4):748-753

19. Ewing DJ, Borsey DQ, Travis P, Bellavere F, Neilson JM, Clarke BF (1983) Abnormalities of ambulatory 24-h heart rate in diabetes mellitus. Diabetes 32(2):101-105

20. Rubenstein JC, Freher M, Kadish A, JJ G (2010) Diurnal heart rate patterns in inappropriate sinus tachycardia. Pacing Clin Electrophysiol 33(8):911-919 